\title{
Direct method for solving second order delay differential equations
}

\begin{abstract}
This paper considers the implementation of fourth order direct method in the form of Adams Moulton method for solving directly second order delay differential equations. The proposed method approximates the solutions using constant step size. Numerical results are presented to show that the proposed code is suitable for solving second order delay differential equations.
\end{abstract}

Keyword: Delay differential equations; Direct method 